\title{
Réflexions sur les limites d'une intégration langues-savoirs disciplinaires entre milieux étrangers
}

\section{Christiane Haeusser}

\section{OpenEdition}

\section{Journals}

Édition électronique

URL : https://journals.openedition.org/asp/3927

DOI : 10.4000/asp.3927

ISSN : 2108-6354

Éditeur

Groupe d'étude et de recherche en anglais de spécialité

\section{Édition imprimée}

Date de publication : 1 décembre 1995

Pagination : 285-301

ISSN : 1246-8185

\section{Référence électronique}

Christiane Haeusser, « Réflexions sur les limites d'une intégration langues-savoirs disciplinaires entre milieux étrangers », ASp [En ligne], 7-10 | 1995, mis en ligne le 01 novembre 2013, consulté le 21 septembre 2021. URL : http://journals.openedition.org/asp/3927 ; DOI : https://doi.org/10.4000/asp. 3927

Ce document a été généré automatiquement le 21 septembre 2021.

Tous droits réservés 


\title{
Réflexions sur les limites d'une intégration langues-savoirs disciplinaires entre milieux étrangers
}

\author{
Christiane Haeusser
}

1 Cette étude s'inscrit dans le cadre des pratiques didactiques qui privilégient diverses formes d'association entre langues et contenus disciplinaires dans l'enseignement des langues aux spécialistes d'autres disciplines. On est tenté de penser que, dans les cas où cette forme d'apprentissage est vécue en milieu naturel, à l'occasion d'échanges interuniversitaires, l'intégration entre savoir-faire langagiers et savoirs disciplinaires devrait se faire de façon optimale. C'est d'ailleurs le terme "cursus intégré » qui est employé pour dénommer les programmes de mobilité étudiante, dans le cadre des échanges Erasmus, qui associent deux ou plusieurs établissements, dans deux ou plusieurs pays et qui conduisent à l'obtention simultanée de deux ou trois diplômes de niveau Bac+4 en fin d'études de second cycle.

2 La multiplication des programmes inter-universitaires, même si tous n'ont pas les mêmes ambitions, mérite que l'on s'interroge sur les conditions et les limites de cette intégration, notamment dans les cas où les contenus et les objectifs de la formation dépassent largement le seul apprentissage de la langue étrangère en milieu naturel - ce que l'on appelle communément le « bain linguistique ». Il faut se rappeler que, lorsque ces programmes ont été conçus, ils reposaient sur l'hypothèse très largement admise des vertus d'une immersion totale, l'exposition constante à langue et culture sous des formes diverses suffisant à elle seule pour faire pénétrer l'une et l'autre, comme par osmose : vision très behaviouriste, quasi pavlovienne de l'apprentissage. 


\section{La situation d'apprentissage}

3 Le cursus intégré sur lequel ont porté la majorité des observations qui vont suivre a été créé en 1987- ce qui permet un certain recul-à partir de formations en langues appliquées et traduction spécialisée, qui associent deux langues étrangères et, pour l'essentiel, trois disciplines d'application: le droit, l'économie, la gestion. On se trouve donc dans la situation relativement complexe où les apprenants sont censés accéder à des savoirs disciplinaires thématiquement assez étendus bien qu'en partie convergents, tout ceci dans trois langues de travail (langue maternelle comprise) et surtout dans trois aires culturelles et trois cadres institutionnels différents.

Plus concrètement, ce cursus, dénommé LEA-Europe, a été conçu pour permettre une rotation géographique des étudiants selon un schéma triangulaire: $1^{\text {re }}$ année dans l'université d'origine, $2^{\mathrm{e}}$ et $3^{\mathrm{e}}$ années successivement dans deux universités d'accueil, Maîtrise réintégrée dans le cursus d'origine. C'est ainsi que les étudiants français effectuent leur $2^{\mathrm{e}}$ année de DEUG en Grande-Bretagne et leur $3^{\mathrm{e}}$ année soit en Allemagne, soit en Espagne, selon la seconde langue étrangère choisie. Ce sont donc deux triangles, partiellement divergents, qui matérialisent les deux itinéraires possibles.

Les enseignements sont approximativement répartis par tiers : un tiers pour chacune des deux langues, un tiers pour les disciplines d'application, dont l'importance va croissant en fin de cursus, ces disciplines constituant le volet professionnalisé de la formation, alors que les deux langues cibles en sont les véhicules obligés dans l'optique d'une orientation vers les affaires internationales.

Dans le cursus d'origine - la branche que nous appelons « sédentaire » (à tort, puisque des séjours à l'étranger, en moyenne de six mois, complètent presque toujours la formation) -, les enseignements de langues s'orientent progressivement vers les langues de spécialité. Les enseignements disciplinaires (droit, économie, économie d'entreprise, puis gestion à partir de la $2^{\mathrm{e}}$ année) sont confiés à des spécialistes de ces disciplines, qui enseignent dans la langue de la communauté linguistique, donc en français aux étudiants francophones. Il s'agit là de la situation maintes fois étudiée où c'est l'enseignant de langues qui va vers la ou les spécialités et non l'inverse.

7 Dans le cursus «itinérant ", l'année initiale est commune avec le cursus d'origine, mais seuls y sont admis, en fin de 1re année, les étudiants qui font preuve d'un bon niveau dans les deux langues cibles et de bases solides en droit et économie générale. À ce stade, seuls les enseignements de civilisation abordent discrètement les discours de spécialité dans les deux langues cibles (institutions, vie économique), sans pour autant les analyser en tant que discours; c'est là aussi que se poursuit la sensibilisation aux différences culturelles, en principe engagée au cours des études secondaires. On peut donc considérer qu'au moment où ils sont transplantés dans la première des deux universités d'accueil, les étudiants forment un groupe assez homogène, possédant une maturité linguistique suffisante pour fonctionner dans la langue cible, mais de façon plus passive qu'active dans un premier temps. En revanche les connaissances disciplinaires sont encore modestes, voire quasi inexistantes dans le cas de la gestion proprement dite.

8 C'est à partir de la $2^{\mathrm{e}}$ année qu'un changement radical se produit : changement de langue communautaire, changement de contexte culturel, de milieu institutionnel et - c'est là ce qui nous intéresse plus spécialement - accès aux savoirs disciplinaires directement dans 
la langue cible, sous des formes qui reflètent des approches didactiques pouvant varier sensiblement d'une institution à l'autre et d'une discipline à l'autre, mais qui ne prennent pas en compte les problèmes de langue en tant que tels.

\section{Les données du problème}

9 On peut tout d'abord se poser un certain nombre de questions du point de vue de la didactique des langues de spécialité. Nous trouvons-nous dans la situation souvent vue comme idéale, où le contexte culturel et linguistique, les documents d'appui et de référence sont véritablement authentiques, en somme dans le type de situation que les enseignants de langues de spécialité tentent de reconstituer, de simuler dans leurs classes ou parfois regrettent de ne pas pouvoir reproduire faute d'une expertise suffisante dans la spécialité ? Ou bien la nécessité d'acquérir simultanément les savoirs et les moyens d'expression, et non pas seulement de nouveaux savoir-faire langagiers appliqués à des savoirs existants, est-elle un facteur qui, partiellement au moins, ralentit ou modifie les processus d'apprentissage?

10 Mais il faut aussi voir le problème sous l'angle des spécialités disciplinaires. Les programmes traités à chacune des étapes de la formation, quelle que soit la langue de travail, étant supposés assimilés en fin de parcours, va-t-on véritablement parvenir à la construction de savoirs disciplinaires cohérents, susceptibles d'être décodés et encodés dans trois langues, y compris bien sûr la langue maternelle? Ou bien la langue par laquelle a été véhiculé un apport de connaissances va-t-elle rester fortement associée à cette partie des acquis et quelles en seront les conséquences?

11 Notre réflexion s'est inspirée d'une part d'un certain nombre d'études en partie applicables à cette situation, quelques-unes assez anciennes, d'autres récemment exposées dans le cadre du GERAS, notamment celle de F. Raby (1994) sur les savoirs linguistiques et savoirs de spécialité. D'autre part, dans une démarche purement empirique, cette réflexion s'est appuyée sur des observations menées auprès de 60 étudiants du cursus qui vient d'être décrit et auprès d'un autre groupe de 24 étudiants tchèques et slovaques reçus en France, spécialistes de droit et d'économie, à titre de comparaison.

12 Trois pôles de recherche, pour s'en tenir aux plus évidents, peuvent fournir des éléments de réponse : les études sur le bilinguisme, sur les contextes linguistiques, les recherches en psycholinguistique (apprentissage et cognition) et les recherches en linguistique et en didactique des langues.

13 1. Se référer à la notion de bilinguisme ne signifie pas que l'on puisse considérer les étudiants d'un tel cursus comme des bilingues potentiels, voire des trilingues au sens strict du terme, pour autant qu'il en existe. Cependant, certains des mécanismes décrits de longue date par des linguistes comme Weinreich (1953), Lambert et l'équipe de Mc Gill au Canada (1963) correspondent assez bien aux situations que nous connaissons. Ainsi Lambert distingue le bilinguisme qu'il appelle " composé », qui s'applique à deux langues apprises dans un même contexte, du bilinguisme dit "coordonné ", qui se développe lorsque "les contextes de l'acquisition de la langue sont séparés culturellement, temporellement ou fonctionnellement " (Lambert, 1963, p.119). Dans ce cas, en tous points comparable à celui de nos étudiants, il se créerait des systèmes distincts, peu perméables entre eux, chaque langue ayant, si l'on se limite à la composante lexicale, son 
système de signifiants couplés à des signifiés et non pas deux signifiants pour un même signifié. C. Noyau (1980), dans une étude comparative entre les « acquisitions guidées » et «non guidées» en milieu naturel, note une grande perméabilité chez certains apprenants, alors que d'autres sont « incapables de traduire d'une langue à l'autre » et pose une question-clé: "Ont-ils deux systèmes sans correspondances autres que référentielles ?» (1980, p.84). D'autres chercheurs, notamment Narcy \& Rebelo (1983) et Narcy (1990), font ressortir les distinctions entre les différents types d'apprenants et leurs stratégies cognitives, qui s'ajoutent aux phénomènes induits par les contextes linguistiques.

14 2. La question des deux systèmes que l'on suppose dans certaines situations de bilinguisme et de la nature des passerelles éventuelles entre ces systèmes n'est pas non plus tranchée par les psycholinguistes. Les recherches se poursuivent sur les structures cognitives à l'œuvre dans l'acquisition et le traitement des différents types de savoirs, sur le rôle respectif des différentes zones du cerveau. Parmi les travaux en cours susceptibles d'étayer la présente étude, on peut citer ceux du CREPCO (1991) sur l'activité inférentielle dans le fonctionnement cognitif, c'est-à-dire le rôle des connaissances acquises et des informations dérivées de l'environnement dans la génération de nouvelles connaissances. Deux chercheurs de ce groupe de recherche (Frenck \& Pynte) travaillent sur la prise en compte de contraintes multilingues dans l'élaboration de modèles d'accès au lexique. Dans l'état actuel des recherches, on voit cependant assez mal comment une situation d'apprentissage aussi complexe que celle qui nous occupe pourrait être véritablement modélisée, vu le nombre de paramètres qui entrent en jeu. Restent les études de terrain : on pourrait, par exemple, analyser les productions des apprenants à la lumière des nombreuses recherches sur les transferts et les interférences, sur les processus de généralisation, sur l'incidence du sens, des images mentales et des modes d'organisation sur la mémorisation; on pense aussi aux stratégies d'apprentissage, dont l'explicitation par les apprenants eux-mêmes pourrait être révélatrice (Atlan, 1993).

15 3. Avant d'aborder les éléments d'analyse que les recherches en linguistique et didactique peuvent fournir, il convient de décrire plus précisément la situation d'apprentissage des étudiants transplantés en milieu étranger. L'apprenant se trouve alors plongé dans le contexte culturel et linguistique de la langue cible qui sera dominante pendant une année universitaire. Il est traité, dans le milieu institutionnel d'accueil, et notamment dans le cadre des enseignements de spécialité, comme un pseudo-natif. Ce sont des économistes, des juristes, des comptables qui enseignent leur discipline, dans leur langue, avec leurs méthodes et un objectif prioritaire : transmettre des savoirs et, dans le meilleur des cas, susciter une réflexion sur ces savoirs. Quant à la langue, elle n'est que le véhicule des savoirs. La formation de ces enseignants, ainsi que le rôle qui leur est assigné, ne les prédisposent pas à un traitement métalinguistique de leur propre discours.

Dans cette situation didactique, on peut considérer que les compétences mises en œuvre par l'apprenant se limitent, pour l'essentiel et dans un premier temps, à comprendre un discours plus ou moins spécialisé, éventuellement comprendre des documents écrits d'accompagnement, les interpréter et mettre au point des techniques qui lui permettront d'en garder la trace en vue de les mémoriser. Dans un second temps seulement, et de façon variable d'un enseignement à l'autre, pourront intervenir d'autres activités langagières telles qu'interaction orale (notamment dans les travaux de groupe), production écrite et orale, toujours centrées sur la réactivation des contenus disciplinaires. Il n'est pas exclu, tous les universitaires le savent, que la première occasion 
de production écrite soit en fait l'examen final. La France n'a pas le monopole de ce type de pratique.

17 L'attitude libérale, non-interventionniste, laisse supposer que le décryptage du discours de spécialité se fait grâce à la fréquence d'écoute et de lecture, par inférence d'après le contexte linguistique et culturel, que l'interprétation sémantique et conceptuelle s'appuie sur les connaissances acquises et que les traces écrites conservées par l'étudiant sont à leur tour compréhensibles et donc utilisables pour étayer la mémorisation. Comme de nombreuses observations incitent à douter, avec J.P. Narcy (1993:268), de l'efficacité de «l'apprentissage sur le tas » à ce niveau de compétence, notamment lorsque les savoirs disciplinaires eux-mêmes sont mal assurés, on doit s'interroger sur d'autres attitudes possibles, en dehors d'une quasi-absence de guidage ou d'un guidage inadapté.

\section{Éléments de l'enquête}

\section{Enquête 1}

La situation didactique décrite, ainsi que les quelques réflexions préalables ont servi de base au questionnaire dont nous allons maintenant commenter les résultats. L'objectif était de mieux comprendre comment est perçu et vécu par les apprenants eux-mêmes l'accès à des savoirs disciplinaires successivement dans trois langues et trois contextes culturels différents. Les langues de travail concernées sont l'anglais, l'allemand, l'espagnol et le français. L'enquête a été menée à trois stades du circuit interuniversitaire, en mars-avril 1995. Les questionnaires étaient anonymes. L'échantillon comprend:

19 - 23 étudiants français de Maîtrise LEA-Europe, qui ont réintégré le cursus d'origine en fin de parcours.

20 - 19 étudiants de $3^{\mathrm{e}}$ année, donc en seconde année de mobilité (étudiants britanniques en France et français en Espagne).

$21-18$ étudiants de $2^{\mathrm{e}}$ année (Allemands et Espagnols au terme de leur séjour en France).

22 La première question porte sur les acquis dans les disciplines de spécialité, préalables au départ à l'étranger. Les étudiants estiment en majorité avoir été formés essentiellement par les enseignements spécialisés de 1re année, dans leur langue. Les enseignements de civilisation étrangère viennent en seconde place. La lecture de la presse, en langue maternelle plus que dans les langues cibles, est citée comme préparation à l'économie, jugée par ailleurs insuffisante dans le cadre institutionnel d'origine par le groupe des Allemands.

Une mention spéciale est faite de deux séminaires organisés à l'Université de Provence : l'un en fin d'année avant la rotation vers l'Angleterre (une initiation au droit anglais, assurée par un collègue venu de Londres) ; l'autre en début de séjour pour les étrangers arrivant en France (une introduction méthodologique et terminologique à l'économie et au droit). La mention spontanée de ces séminaires mérite attention : on peut penser qu'ils répondent en partie aux besoins de passerelles entre différentes approches didactiques et différentes langues, mais aussi à un besoin de traitement métalinguistique des discours spécialisés, qui fait défaut par la suite, lorsque les étrangers sont noyés parmi les natifs et hésitent à poser des questions dont ils craignent qu'elles n'intéressent pas le reste de l'auditoire. 

quelques remarques sur « des phrases longues et compliquées ». L'enquête ne permet pas de vérifier si certains problèmes de compréhension orale sont en fait d'ordre syntaxique, liés à des difficultés de perception de l'organisation des énoncés : une question sur la compréhension écrite aurait peut-être permis de faire mieux ressortir les problèmes relatifs à cette composante.

31 Dans la rubrique « autres » apparaissent surtout des problèmes d'ordre méthodologique : «la façon d'aborder la matière», "la technique de la dissertation économique ». Certaines inquiétudes relatives aux examens portent beaucoup moins sur l'acquisition des connaissances que sur la façon de les retraiter, de les relier aux documents, de les présenter: "on ne sait pas comment utiliser les documents pour répondre aux questions », « on réfléchit plus lentement ». 
Les propositions quant aux moyens de surmonter les difficultés sont dispersées du fait que la question était très ouverte. Elles peuvent être regroupées en trois grandes rubriques :

1. Renforcer les acquis dans chaque discipline avant la période d'expatriation : cours spécialisés en langue maternelle, lectures guidées, « apprendre le vocabulaire de base par spécialité ».

2. Multiplier les contacts périphériques aux enseignements de spécialités. La forme existante des enseignements étant vue comme immuable parce que liée aux traditions du milieu institutionnel, ce sont des moyens annexes qui sont suggérés, fortement marqués par les besoins de contacts plus personnalisés : contacts informels avec les enseignants ; séances de reprise systématique et contrôle de la prise de notes; travail parallèle en cours de langues (traductions spécialisées, expression) ; groupes de travail avec des natifs. Le besoin d'un guidage venant en complément des enseignements disciplinaires, faute de pouvoir les accompagner directement, apparaît très clairement.

3. Faciliter l'adaptation aux nouvelles approches didactiques : explicitation des méthodes et objectifs par les spécialistes eux-mêmes, entraînement aux techniques de lecture et de traitement des documents, points de presse pour guider l'accès à l'information.

Les moyens extra-universitaires invoqués pour contribuer à l'acquisition des savoirs disciplinaires et des langues de spécialités sont pour l'essentiel les stages en entreprise et les emplois temporaires. En période universitaire, ils se limitent à la presse, la radio, quelques rares contacts avec des spécialistes. On peut craindre que ce ne soit là le signe d'une excessive concentration sur les tâches universitaires, au détriment des recherches personnelles.

7 Les trois dernières questions posent directement le problème de la perméabilité des acquis disciplinaires et linguistiques entre milieux étrangers.

Tous les sujets sans exception estiment fonctionner à l'intérieur de systèmes distincts, où langue et savoir disciplinaire sont fortement associés: "même une fois comprises les correspondances avec le français, je continue à utiliser les termes anglais ou espagnols selon les matières, dans mes notes et fiches personnelles »; « je continue à raisonner dans la langue dans laquelle la matière a été apprise »; «je ne fais pas de transferts entre langues ». Ce phénomène est accentué lorsque la discipline n'a pas été préalablement abordée (cas de la gestion). Une étudiante de maîtrise, tutrice d'un groupe d'Allemands, dit avoir découvert des équivalents français de termes de gestion appris en Allemagne en travaillant avec son groupe de tutorat.

Le recours à la traduction, aux dictionnaires ne se pratique qu'en cas de nécessité, de difficulté, de confusion. Plusieurs étudiants de maîtrise se sont rendu compte progressivement, "après coup ", que "les termes sont les mêmes ", que " beaucoup de mots sont transparents ». Le lexique n'est donc pas ressenti comme un obstacle majeur.

La question de l'intégration des acquis disciplinaires en fin de cursus n'a été posée qu'au groupe de maîtrise : la plupart insistent sur le temps nécessaire pour intégrer au retour les connaissances acquises dans l'une des langues cibles, là où la progression dans la discipline l'exige : «j'ai du mal à reconstruire mes connaissances en français ». Plusieurs d'entre eux (5 sur 23) estiment qu'ils ont dû réapprendre certaines notions dans leur langue. Dans un certain sens, la langue maternelle devient une langue étrangère dans laquelle il faut retraiter les savoirs acquis. D'autres soulignent le manque de continuité, de cohérence dans la progression, l'oubli rapide des connaissances acquises en langue 
cible lorsqu'elles ne sont pas réactivées. Ils craignent d'avoir des lacunes, des connaissances moins approfondies que le groupe du cursus d'origine. Ils invoquent aussi les différences d'approches et de contextes culturels : «les disciplines sont abordées de façon trop spécifique au pays pour être facilement réutilisées ».

Enfin les réponses à la question sur les avantages-inconvénients du système, posée à tous, sont globalement très favorables à ce type de cursus, ce qui peut paraître surprenant au vu des jugements sévères qui ont été rapportés. Mais en fait ce sont avant tout le niveau atteint en langues et l'étendue des savoir-faire langagiers qui donnent satisfaction: aisance à l'oral, "pas de problème pour discuter dans les deux langues, même avec des spécialistes, des professionnels ", " on pense en langue étrangère ", " on lit facilement, même des ouvrages spécialisés », « le vocabulaire spécialisé est appris dans son contexte ». Trois étudiants de maîtrise soulignent une bonne aptitude à différencier registre formel et informel. Ce point mériterait d'être vérifié par une analyse des discours produits, car il semble en contradiction avec certaines critiques, notamment des enseignants d'anglais qui ont noté la tendance de ces étudiants à faire un usage abusif de la langue parlée dans leurs mémoires de fin d'études. Du point de vue des savoirs disciplinaires, les jugements sont beaucoup plus réservés. Les difficultés sont particulièrement sensibles à chaque nouvelle rotation: "au début on perd de l'information », « les connaissances sont plus approximatives ». En revanche, un bon tiers des sujets apprécient la capacité d'adaptation aux différences culturelles, une familiarisation avec des méthodes, des systèmes différents.

C'est donc essentiellement la progression dans les savoirs disciplinaires qui ne répond pas aux attentes : les blocages se situent d'abord à la source, dès le stade de la compréhension du discours de spécialité, lorsqu'il est livré sans mode d'emploi ; ils se manifestent ensuite lorsque les morceaux du puzzle restent dispersés, faute d'une véritable intégration centralisée des connaissances reçues dans les trois langues. La première impression qui se dégage de cette partie de l'enquête est que l'enseignement des savoirs disciplinaires en milieu exolingue, tel qu'il est largement pratiqué dans ce type de programme universitaire, profite beaucoup plus à l'enrichissement des compétences en langues qu'à l'acquisition des savoirs eux-mêmes.

\section{Enquête 2}

43 L'autre volet de l'enquête, menée au moyen du même questionnaire auprès de 24 étudiants tchèques et slovaques, en majorité économistes ou juristes, à l'issue d'un semestre d'études en France dans le cadre d'un programme Tempus, a donné lieu à certaines réponses sensiblement différentes, d'autres très concordantes. La comparaison est malaisée dans la mesure où les conditions des deux expériences n'étaient pas identiques, mais les différences de réactions nous ont paru refléter précisément les écarts avec la situation d'apprentissage précédemment décrite, qui portent pour l'essentiel sur : le domaine de spécialité limité à une discipline; un bon niveau d'expertise dans la discipline de spécialité ; une seule langue cible ; des mesures d'accompagnement mises en place avec des enseignants de français de spécialité. Nous ne ferons ici que mentionner les remarques directement liées à notre propos, sans reprendre les éléments du questionnaire.

44 Pour faciliter l'accès au discours spécialisé en langue cible, ces étudiants s'appuient fortement sur les acquis disciplinaires, mais aussi sur le lexique spécialisé, que ce soit la 
terminologie déjà abordée en langue de spécialité dans l'université d'origine ou des mots transparents, plus nombreux qu'on ne pourrait le supposer dans le cas des langues slaves, et des disciplines concernées. Encore faut-il que l'étudiant identifie les transparences pour que la compréhension puisse être étayée par ce type de repères lexicaux. Trois étudiants soulignent les différences entre langue commune et « langue universitaire ». Le fait que les seuls sujets qui aient été sensibles à cette différence de registre appartiennent tous à ce groupe peut sans doute s'expliquer par le phénomène de diglossie langue commune/langue littéraire, assez marqué en tchèque et slovaque.

Le travail d'accompagnement expérimenté avec ce groupe semble avoir bien fonctionné : un enseignant de français a assisté aux premiers cours d'économie et de droit en utilisant avec eux une grille d'écoute où ont été notés les indices d'énonciation (pronoms, verbes d'opinion), les indices d'organisation (connecteurs et équivalents tels que «ceci fera l'objet de», etc.) et le lexique spécialisé. Ces grilles ont ensuite été commentées et comparées pour faire ressortir constantes et variantes d'un enseignant à l'autre dans une même spécialité et d'une spécialité à l'autre. Une technique similaire a été appliquée à la lecture de textes spécialisés pour le repérage des articulations du discours, de l'ordre des constituants (exemples connus : la fréquence, dans la langue juridique, des circonstants en début de phrase, des relatives en incises). Il en ressort, pour résumer les commentaires, une amélioration de la précision et de la vitesse de compréhension, une meilleure organisation de la prise de notes, donc de meilleures bases en vue de la mémorisation.

Plus de la moitié des sujets ont noté l'ouverture culturelle et disciplinaire vers de «nouvelles applications", de "nouveaux systèmes", tout particulièrement les économistes qui ont dû reconsidérer leur discipline dans la perspective d'une économie de marché. Cependant, l'absence de toute remarque, dans ce groupe, sur des lenteurs ou des restrictions dans l'adaptation aux savoirs disciplinaires nous paraît confirmer le gain d'efficacité lorsque la spécialité est mieux maîtrisée avant la période d'expatriation. Ce ne sont pas les capacités langagières qui pouvaient être déterminantes, les deux groupes étant de niveau comparable, malgré une plus grande hétérogénéité dans le groupe Tempus.

47 Les deux groupes de sujets, cursus intégré et Tempus, se rejoignent sur un point qui nous paraît essentiel et qui méritera de plus amples observations : du point de vue des langues de travail utilisées dans le cadre des enseignements de spécialités, il semble bien que se crée une situation de bilinguisme du type "coordonné ", impliquant le fonctionnement de deux systèmes distincts, relativement étanches. Des remarques telles que « on pense et on travaille les cours tout en français ", " on discute en français de ce qu'on a appris en français, même entre nous" vont dans le sens de celles que nous avons relevées précédemment. Ceci n'a rien de surprenant, on peut même penser que c'est là le but recherché. Mais il reste à se demander quelle est l'incidence de l'association langue/ savoir sur l'intégration des nouveaux savoirs disciplinaires dans le système de pensée, de références, de construction des concepts et des connaissances.

On ne peut évidemment donner qu'une valeur indicative aux résultats d'une enquête sommaire qui reflète surtout les intuitions des apprenants. Ces premières informations nous paraissent cependant exploitables dans deux directions. Elles peuvent d'une part ouvrir une réflexion sur les pratiques actuelles et inciter à reconsidérer certaines des modalités de ce type de programmes inter-universitaires, au vu des observations les plus 
récurrentes. Elles devraient d'autre part permettre de déterminer dans quel sens et sous quelle forme poursuivre les recherches au-delà de ce premier stade purement empirique.

\section{Les prérequis}

49

a généralement été la langue du pays d'accueil, autrement dit un sociologue reçu dans une université allemande aura-t-il les capacités langagières nécessaires pour communiquer dans son domaine de spécialité en allemand? Si tel n'est pas le cas, une mise à niveau est envisagée et peut même faire l'objet d'un financement spécifique. Rien de comparable n'a été prévu pour les spécialistes de langues, qui sont censés posséder, de par leur formation, les prérequis linguistico-culturels, donc des outils directement liés aux connaissances à acquérir. Mais rien n'a été prévu non plus pour les étudiants en situation intermédiaire, dont la polyvalence même limite nécessairement, au moins en début de formation, le degré d'expertise dans chacune des disciplines: langues (tout particulièrement dans leurs applications aux spécialités) et disciplines spécialisées. On n'est ni dans la situation où la spécialité est suffisamment maîtrisée pour servir de support aux moyens d'expression, ni dans la situation inverse où la langue est suffisamment maîtrisée pour véhiculer parfaitement l'acquisition des connaissances nouvelles.

50

Faut-il pour autant concevoir des cursus plurilingues qui se borneraient à une reprise des mêmes contenus, successivement dans deux langues cibles, la progression dans les savoirs disciplinaires étant alors négligée au profit des langues? Une première expérience dans ce sens nous a appris que la répétition démotive dès que l'obstacle de la langue est surmonté. Si donc il est préférable, et souhaitable en fonction des objectifs de la formation, de développer les connaissances dans des disciplines autres que les langues, c'est le problème des prérequis qui devra être traité en priorité, à chaque stade du cursus, et plus particulièrement dans les disciplines qui exigent des savoirs très construits, des cadres de références théoriques, par exemple l'économie. Quand on sait combien ce problème est difficile à résoudre dans n'importe quel cursus universitaire, on mesure aisément l'ampleur de la tâche lorsque plusieurs institutions doivent collaborer, chacune avec ses structures et ses cursus existants, sans compter les différences de culture institutionnelle et disciplinaire. Là où une trop grande discontinuité, dans les contenus ou dans les méthodes, risque de ralentir ou de fausser la mise en place de nouveaux concepts et de nouvelles connaissances, il serait tout à fait justifié d'introduire des relais adaptés à la spécialité, tout comme les spécialistes bénéficient d'un soutien en langues avant la période d'études à l'étranger.

51 L'introduction méthodologique et terminologique, que nous avons proposée aux étudiants étrangers à leur arrivée en France pour les préparer aux enseignements d'économie et de droit, a joué un double rôle : familiariser avec les pratiques didactiques et les discours de spécialité, mais aussi vérifier les acquis supposés et identifier les lacunes ou les divergences de contenus. Il est regrettable que l'insuffisance de moyens financiers, parfois de formateurs qualifiés, limite ces enseignements de soutien. 


\section{Les pratiques didactiques}

l'absence ou l'insuffisance d'activité métalinguistique appropriée, de dynamique interactive, de traitement guidé de l'information qui nous parait limiter les effets bénéfiques de l'immersion en milieu institutionnel exolingue. On retrouve là certaines des causes d'échec maintes fois observées dans les situations d'apprentissage en autonomie non guidée. L'enquête laisse supposer que des stratégies d'apprentissage palliatives se mettent en place, mais de façon désordonnée, peu efficace. On peut s'interroger par exemple sur les notions qui s'installent derrière les mots en l'absence de tout recours aux dictionnaires (ceci renvoie à l'enquête), en l'absence aussi d'explicitation des problèmes de compréhension, sous forme de définitions, de paraphrases, de réemplois (pratiques qui, le plus souvent, accompagnent un cours de langue de spécialité ou de spécialité en langue cible). On peut aussi se demander si des formes ou des notions erronées n'ont pas tendance à se fossiliser, faute de correction des erreurs.

S'il n'est pas possible, et sans doute pas souhaitable, d'offrir aux étudiants non natifs des enseignements de spécialité "sur mesure », on peut toutefois proposer des substituts. Là où les moyens financiers le permettent, quelques séances de synthèse avec les spécialistes, hors séances plénières, suffisent pour mieux cerner les méthodes, les sousentendus disciplinaires et les objectifs à atteindre, et aussi pour canaliser le travail personnel. 

linguistique des cours de droit et d'économie par un enseignant de langue de spécialité, expérimentée avec le groupe Tempus, a bien rempli cette fonction. Le coût d'un travail en binôme spécialiste/enseignant de langue est cependant tel que l'expérience a dû être limitée à quelques séances. Parmi les enseignements de langues traditionnels, les cours de traduction paraissent les plus aptes à servir de relais aux disciplines de spécialité, par une approche linguistique des problèmes de traduction, par des études terminologiques, par exemple. Tout dépend du degré de spécialisation des enseignants-traducteurs et de leur volonté de concertation avec les spécialistes.

L'entraînement à la prise de notes, déjà mentionné dans l'enquête, mérite une attention particulière. Les contrôles effectués, sur notre demande, par les tuteurs affectés aux groupes d'étudiants étrangers, ont permis de déceler des distorsions considérables dues à un mauvais calque de l'entendu, d'où des interprétations erronées et des reproductions pour le moins incongrues. Or, en l'absence de documents d'appui écrits, la prise de notes est le seul intermédiaire possible entre le message reçu et l'acte volontaire de mise en mémoire, au moins dans les cas - et ils sont nombreux - où les connaissances à acquérir ne passent pas par une pratique qui permet une réactivation, des reformulations des contenus (telle que les études de cas). Là encore, l'autonomie totale présente des dangers, ne serait-ce que celui d'une mémorisation figée sous une seule forme d'expression en langue cible, apprise par cœur et privée de sa véritable dimension sémantique. On peut craindre que ce mode d'apprentissage ne soit l'une des causes des difficultés d'intégration transversale des savoirs disciplinaires.

Enfin, pour les disciplines qui se prêtent aux applications, comme la gestion, on peut recourir au travail en groupes mixtes natifs/non-natifs, qui favorise l'interactivité, les réemplois, l'élucidation des concepts. Ceci a également l'avantage d'amorcer les contacts informels avec les pairs, parfois longs à se mettre en place.

\section{Perspectives de recherche}

Les directions dans lesquelles pourraient se poursuivre ces recherches découlent pour partie des résultats des premières enquêtes et pour partie des observations des enseignants sur les productions des étudiants francophones en fin de parcours interuniversitaire, donc au moment du bilan de l'expérience.

Les remarques convergent sur la nature des savoir-faire langagiers: aisance dans le maniement de la langue en tant qu'outil de communication orale, y compris dans les applications aux spécialités; rapidité de compréhension globale des textes. Les enseignants de langues ont pour leur part noté, dans les travaux écrits, une tendance à adopter un registre proche de la langue orale, avec quelques erreurs très récurrentes, qui pourraient être les manifestations d'une interlangue fortement fossilisée. Il paraitt possible d'étudier ces modes de fonctionnement en procédant à une analyse systématique d'échantillons des différents travaux réalisés en Maîtrise : exposés, dossiers, mémoires et rapports de stage, dans les deux langues cibles. Les productions de ces étudiants peuvent en outre faire l'objet d'une comparaison avec celles des étudiants de la filière d'origine, soumis aux mêmes travaux.

61 On trouve également des avis concordants sur les domaines de spécialité : notions approximatives, morcellement des connaissances, compensés par des capacités 
d'assimilation et d'adaptation très rapides. Il faut ici faire la part des carences dans l'harmonisation des contenus disciplinaires entre les différentes institutions partenaires et celle des blocages cognitifs dans la construction des savoirs : peut-on voir là les effets d'une très forte association entre les savoirs et la langue qui les a véhiculés, ou bien les répercussions sur l'activité mentale d'une maîtrise imparfaite de la langue de travail (on reproduit plus qu'on ne construit une réflexion)? Si les dysfonctionnements d'origine institutionnelle sont relativement faciles à déceler, il n'en est pas de même pour tout ce qui touche aux processus cognitifs qui entrent en jeu, aux systèmes intermédiaires qui se créent dans des situations d'apprentissage multilingues aussi complexes. C'est là un domaine qui relève de la psychologie cognitive et des études sur le bilinguisme et le plurilinguisme.

Ni l'enquête, ni les enseignants n'ont mentionné de problèmes d'interférences entre les deux langues cibles, auxquels on aurait pu s'attendre dans cette situation d'apprentissage. L'analyse des productions de ces apprenants que nous nous prévue pourra peut-être nous éclairer sur ce point également. Il serait intéressant de rechercher si la compartimentation des savoirs, observée par ailleurs, va de pair avec une certaine étanchéité entre les langues pratiquées dans des contextes culturels et institutionnels distincts.

\section{Conclusion}

Après l'enthousiasme initial pour les programmes d'échanges européens, il faudrait maintenant passer à une seconde phase où une meilleure conscience des conditions d'apprentissage en milieu étranger permettrait de guider utilement les apprenants. Le véritable défi est de trouver l'équilibre entre le besoin de renforcer la langue cible en la faisant fonctionner de façon spontanée et authentique et la nécessité de renvoyer aux cadres référentiels déjà établis et véhiculés par la langue maternelle, afin d'installer correctement et durablement les nouvelles connaissances. La tendance à considérer les étudiants étrangers comme des pseudo-natifs est peut-être un moyen facile de se cacher les résistances institutionnelles et disciplinaires à une véritable évolution vers la dimension européenne.

\section{BIBLIOGRAPHIE}

Atlan, J. 1993. «Stratégies utilisées par des étudiants pour présenter un court texte technique ». ASp 2, 121-137.

Frenck, C. \& J. Pynte. 1991. Centre de recherche en psychologie cognitive (CREPCO). Rapport d'activité CNRS.

Lambert, W. 1963. "Psychological approaches to the study of language ». The Modern Language Journal 67/3, 114-121. 
Narcy, J.P. \& M. Rebelo. 1983. « What happens to LSP when students take the initiative ». In Perrin, M. (dir.), Pratiques d'aujourd'hui et besoins de demain, Actes du 4 e Symposium européen sur les langues de spécialité. Bordeaux : Université Bordeaux 2, 81-94.

Narcy, J.-P. 1990. Apprendre une langue étrangère. Paris : Éditions d'organisation.

Narcy J.-P. 1993. « Approche nouvelle de la grammaire ». ASp 2, 259-272.

Noyau, C. 1980. «Étudier l'acquisition d'une langue non-maternelle en milieu naturel ». Langages $57,73-86$.

Raby, F. 1994. «Les relations entre savoirs linguistiques et savoirs de spécialité dans la construction des processus de passage de l'être 'cultivé' à l'être 'civiliséc ». ASp 5-6, 121-130.

Weinreich, U. 1974 [1953]. Languages in Contact. La Hague: Mouton. 1974.

\section{RÉSUMÉS}

Dans le cadre des programmes européens, les étudiants suivent les enseignements de leur spécialité directement en langue étrangère dans le contexte institutionnel du pays d'accueil. Pour ceux dont la formation associe une, voire deux langues cibles et des disciplines d'application telles que l'économie et le droit, dans lesquelles leur degré d'expertise est encore modeste, la question des modes d'interaction entre savoir-faire langagiers et savoirs disciplinaires, des processus cognitifs qui entrent en jeu, se présente de façon particulièrement complexe. Une première enquête fait ressortir une forte association entre les savoirs disciplinaires et les langues qui les ont véhiculés, un manque de perméabilité inter-langues et une difficulté à réintégrer les acquis dans le contexte de la langue maternelle.

In the framework of European programmes, students study their specialized subjects in the language and in the institutional context of the host country. For those whose curriculum includes one or even two foreign languages together with applied subjects such as economics and law, in which their expertise is still limited, the problem of a possible interaction between their language skills and their acquisitions in the applied subjects, and the problem of the cognitive processes which come into play, are particularly difficult to identify. A preliminary survey shows a close connection between their knowledge in the disciplines and the languages in which they were studied, very little cross-transfer between the languages and difficulty in using the acquired knowledge in the relevant context in their mother tongue.

\section{INDEX}

Keywords : bilingualism, culture, learning, LSP, pluridisciplinarity

Mots-clés : apprentissage, bilinguisme, culture, langue de spécialité, pluridisciplinarité

\section{AUTEUR}

\section{CHRISTIANE HAEUSSER}

Christiane Haeusser enseigne en filière LEA à l'Université de Provence. Elle est l'auteur d'une thèse de $3^{\mathrm{e}}$ cycle, « Les difficultés lexicales dans l'apprentissage de l'anglais par les 
francophones ", Université de Provence (1981) et, en collaboration, de la série des ouvrages pédagogiques CLEAR, aux éditions Martorana. christiane.haeusser@univ-aix.fr 